\title{
YAP regulates porcine skin-derived stem cells self-renewal partly by repressing Wnt/ $\beta$-catenin signaling pathway
}

\author{
Hong-Chen Yan ${ }^{1} \cdot$ Yu Sun ${ }^{1} \cdot$ Ming-Yu Zhang ${ }^{1} \cdot$ Shu-Er Zhang ${ }^{2} \cdot$ Jia-Dong Sun ${ }^{1} \cdot$ Paul W. Dyce ${ }^{3}$. \\ Francesca Gioia Klinger ${ }^{4}$. Massimo De Felici ${ }^{4}$. Wei Shen ${ }^{1}$. Shun-Feng Cheng ${ }^{1}$ (i)
}

Accepted: 9 September 2021

(c) The Author(s), under exclusive licence to Springer-Verlag GmbH Germany, part of Springer Nature 2021

\begin{abstract}
Skin-derived stem cells (SDSCs) are a class of adult stem cells (ASCs) that have the ability to self-renew and differentiate. The regulation mechanisms involved in the differentiation of SDSCs are a hot topic. In this paper, we explore the link between the transcriptional regulator yes-associated protein (YAP) and the fate of porcine SDSCs (pSDSCs). We found that lysophosphatidylcholine (LPC) activates YAP, promotes pSDSCs pluripotency, and counteracts transdifferentiation of pSDSCs into porcine primordial germ cell-like cells (pPGCLCs). YAP promotes the pluripotent state of pSDSCs by maintaining the high expression of the pluripotency genes Oct 4 and Sox2. The overexpression of YAP prevented the differentiation of pSDSCs, and the depletion of YAP by small interfering RNA (siRNAs) suppressed the self-renewal of pSDSCs. In addition, we found that YAP regulates the fate of pSDSCs through a mechanism related to the Wnt/ $\beta$-catenin signaling pathway. When an activator of the Wnt/ $\beta$-catenin signaling pathway, CHIR99021, was added to pSDSCs overexpressing YAP, the ability of pSDSCs to differentiate was partially restored. Conversely, when XAV939, an inhibitor of the Wnt/ $\beta$-catenin signaling pathway, was added to YAP knockdown pSDSCs a higher self-renewal ability resulted. Taken together, our results suggested that YAP and the Wnt/ $\beta$-catenin signaling pathway interact to regulate the fate of pSDSCs.
\end{abstract}

Keywords pSDSCs $\cdot$ YAP $\cdot \beta$-catenin $\cdot$ Wnt pathway $\cdot$ Self-renewal

\section{Introduction}

Embryonic stem cells (ESCs) and adult stem cells (ASCs) have the ability to self-renew and differentiate (Mo et al. 2014a). Past studies have shown that ESCs and/or ASCs derived from human, mouse, and pig have the capacity to differentiate into germ cells, oocyte-like cells (OLCs), and even produced offspring (Dyce et al. 2006; Ge et al. 2015;

Wei Shen

wshen@qau.edu.cn; shenwei427@163.com

Shun-Feng Cheng

sfcheng@qau.edu.cn

1 College of Life Sciences, Institute of Reproductive Sciences, Qingdao Agricultural University, Qingdao 266109, China

2 Animal Husbandry General Station of Shandong Province, Jinan 250010, China

3 Department of Animal Sciences, Auburn University, Auburn, AL 36849, USA

4 Department of Biomedicine and Prevention, University of Rome Tor Vergata, 00133 Rome, Italy
Hayashi et al. 2012). Fetal skin harbors populations of stem cells responsible for development and maintenance into adulthood, making skin an attractive source for ASCs $(\mathrm{Ge}$ et al. 2016). As a result of ethical considerations human embryos are not accessible for research, resulting in the use of porcine models. Porcine models have close similarities to humans with respect to their genetic relationship and the onset of epigenetic programs, making them ideal animal models for investigating human biology (Kobayashi et al. 2017). Porcine skin-derived stem cells (pSDSCs) offer an ideal in vitro model to investigate human ASCs (Yan et al. 2019). During last 10 years, germ cells differentiated from pSDSCs have been extensively investigated (Ge et al. 2016; Dyce et al. 2006; Yan et al. 2019). But the efficiency of pSDSCs differentiation into porcine primordial germ celllike cells (pPGCLCs) was very low (Linher et al. 2009; Yan et al. 2019). This problem can be solved by improving the differentiation efficiency or generating large amounts of pSDSCs through enhancing self-renewal. Recent studies have suggested that YAP is required for the efficient 
self-renewal of stem cells (Qin et al. 2016; Mo et al. 2014b; Totaro et al. 2017).

The Hippo signaling pathway has been widely studied in mammalian cells and animal models (Singh and Mia 2019; Jeong et al. 2018). Hippo signaling regulates diverse cellular processes including cell proliferation and differentiation (Yang et al. 2017). In mammals, The core components of the Hippo pathway were mammalian Ste2-like kinases (MST1/2, Hpo orthologs) and large tumor suppressor kinase 1/2 (Lats 1/2, Wts orthologs). Activation of Hippo signaling results in YAP being phosphorylated, sequestered to the cytoplasm, and degraded (Seo et al. 2013). Recently, emerging evidence suggests that YAP is a crucial regulator for maintaining the self-renewal of ESCs, induced pluripotent stem cells (iPSCs), and ASCs in vitro and in vivo (Tamm et al. 2011; Lian et al. 2010; Mo et al. 2014b; Qin et al. 2016; Ramalho-Santos et al. 2002). Additionally, YAP also regulates self-renewal of tissue-specific progenitor cells, such as skin epidermal progenitor cells, cardiac progenitor cells, muscle progenitor cells, and neural progenitor cells (Fuchs and Chen 2013; Lee et al. 2008; Xin et al. 2013; Judson et al. 2012). For instance, Zhang et al. (2011) found that activation of the transcriptional auxiliary activity of epidermal YAP in mice can inhibit their terminal differentiation leading to the expansion of epidermal progenitor cells in the basement membrane.

Canonical Wnt signaling is a highly conserved pathway (Murillo-Garzón and Kypta 2017) that is dependent on $\beta$-catenin whose role is primarily based on whether $\beta$-catenin is stable in the cytoplasm or translocated to the nucleus. In the basic state, $\beta$-catenin in the cytoplasm is mainly inhibited by GSK3 $\beta$, which holds Wnt signaling in an inhibited state (Murillo-Garzón and Kypta 2017; Huang et al. 2009). It was found that there is a multipoint cross correlation between the Wnt and Hippo signaling pathways (Piccolo et al. 2014). The interaction between Hippo signaling and Wnt signaling lies in the mutual regulation of their core molecules. The Hippo signaling pathway can affect the expression of Wnt target genes by changing the transcriptional activity of $\beta$-catenin. Qin et al. (2016) reported that YAP-overexpressing human embryonic stem cells (hESCs) display lower levels of active $\beta$-catenin and Wnt target genes. Zhao et al. (2011) found that YAP can interact with $\beta$-catenin and induce the expression of Wnt target genes in mouse heart tissue. These studies confirmed that Hippo and Wnt signaling indeed regulate each other's activity.

Self-renewal is a critical aspect of stem cells allowing them to increase in number while maintaining pluripotency (Fuchs and Chen 2013). The molecular mechanisms involved in maintaining the self-renewal of ESCs and ASCs in vitro have been a key issue in stem cell research. Emerging evidence has indicated that YAP may play an important role in maintaining the pluripotency of stem cells (Qin et al. 2016;
Zhao et al. 2011; Mo et al. 2014b). To date, reports concerning the influence of YAP on the pluripotency of ASCs are limited. Researchers suggested that the Hippo signaling pathway plays different roles in different kinds of stem cells, either being inhibitory or activating, depending on biological contexts (Mo et al. 2014b; Imajo et al. 2012; Yang et al. 2017; Zhao et al. 2011). To date, studies concerning the role of YAP in ASCs are limited, and the mechanisms of its influence on self-renewal and differentiation of ASCs remain unclear. In this study, we used the pSDSCs to explore the effect of YAP on ASC self-renewal. Our data suggested that the upregulation of YAP contributes to the maintenance and self-renewal capacity of pSDSCs by maintaining the high expression of the pluripotency genes Sox 2 and Oct 4 and meanwhile suppressing Wnt signaling.

\section{Materials and methods}

\section{pSDSCs isolation, culture, and differentiation}

Pigs were housed in Laixi, Shandong province. This research was approved by the Ethics Committee of Qingdao Agriculture University (Agreement No. 2019-011).

Porcine back skin tissue was collected from day 40 to 45 gestation fetus (E40-45). The isolation, culture, and differentiation of pSDSCs were described previously (Fig. 1a) (Yan et al. 2019; Dyce et al. 2006). Briefly, the porcine skin tissue was mechanically dissociated using a pipette. The pSDSCs were incubated in SDSCs medium (Yan et al. 2019). After 12 days culture, the cells are positive for Oct4, Sox2, and YAP (Fig. 1). These cells could differentiate into pPGCLCs under in vitro differentiation conditions (Yan et al. 2019). Briefly, the pSDSC colonies were disaggregated into single cells and $1 \times 10^{5}$ cells were seeded into $60-\mathrm{mm}$ tissue culture dishes within differentiation medium (Yan et al. 2019). Every 2 days the differentiation medium was changed. At approximately 20 days cells with the morphological characteristics of pPGCLCs were present.

\section{Indirect immunofluorescence assay (IFA)}

Expression of Nanog, Oct4, Sox2, and YAP in pSDSCs was examined by IFA. Meanlwhile, we have confirmed that all the antibodies used in this paper are applicable in the pig. After 12 days culture, the cell colonies which named pSDSCs were collected (Fig. 1a) and fixed in $4 \%$ paraformaldehyde for $30 \mathrm{~min}$; then the samples were transferred to microscope slides treated with poly-L-lysine. The samples were permeabilized with $0.5 \%$ Triton-X-100 for $10 \mathrm{~min}$ and then blocked with $10 \%$ bovine serum albumin overnight at $4{ }^{\circ} \mathrm{C}$. The pSDSCs samples were covered with $50 \mu 1$ primary antibody (Table $\mathrm{S} 1$ in the supplementary 
Fig. 1 Culture and characterization of porcine skin-derived stem cells (pSDSCs). a Morphology of pSDSCs at different culture stages. Separation and culturing of pSDSCs and the process of passaging. Scale bar $=100 \mu \mathrm{m}$. b Immunofluorescence with Sox2, Oct4, Nanog, and YAP antibodies (red) and Hoechst33342 (blue) of pSDSCs. Scale bar $=10 \mu \mathrm{m}$. c Flow cytometry analysis of Sox2, Oct 4 , and YAP in pSDSCs. $\mathbf{d}$ Representative western blot (WB) and relative densitometric analysis of Sox2, Oct4, and YAP expression in pSDSCs at different stages. The results are presented as mean $\pm \mathrm{SD}$. $* P<0.05 ; * * P<0.01$

a

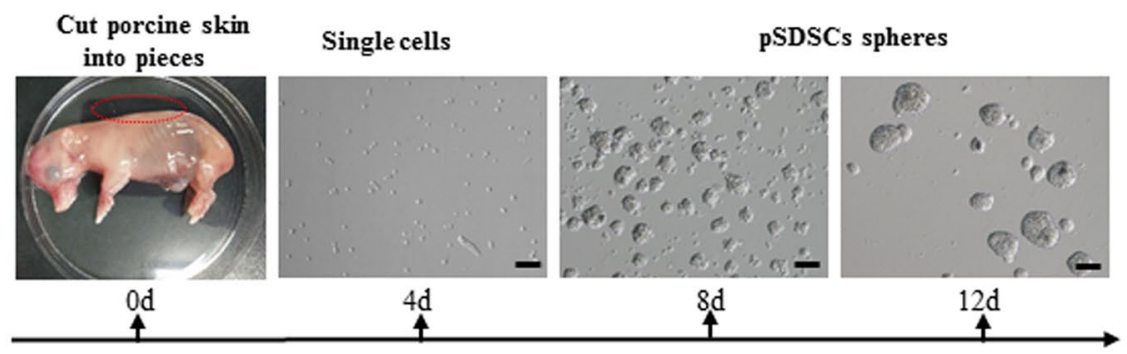

b
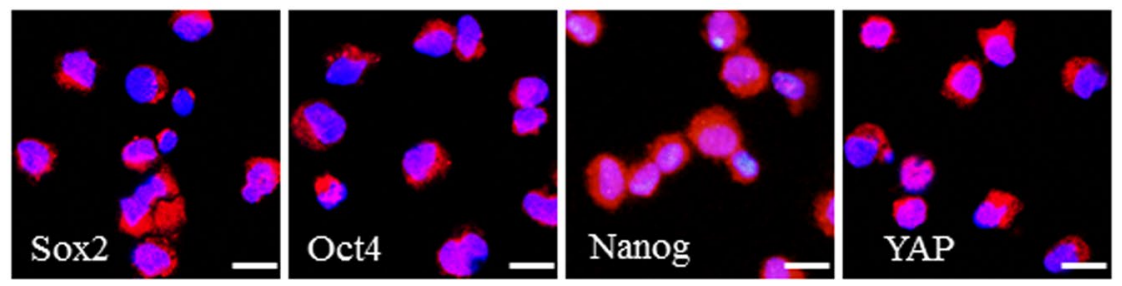

c

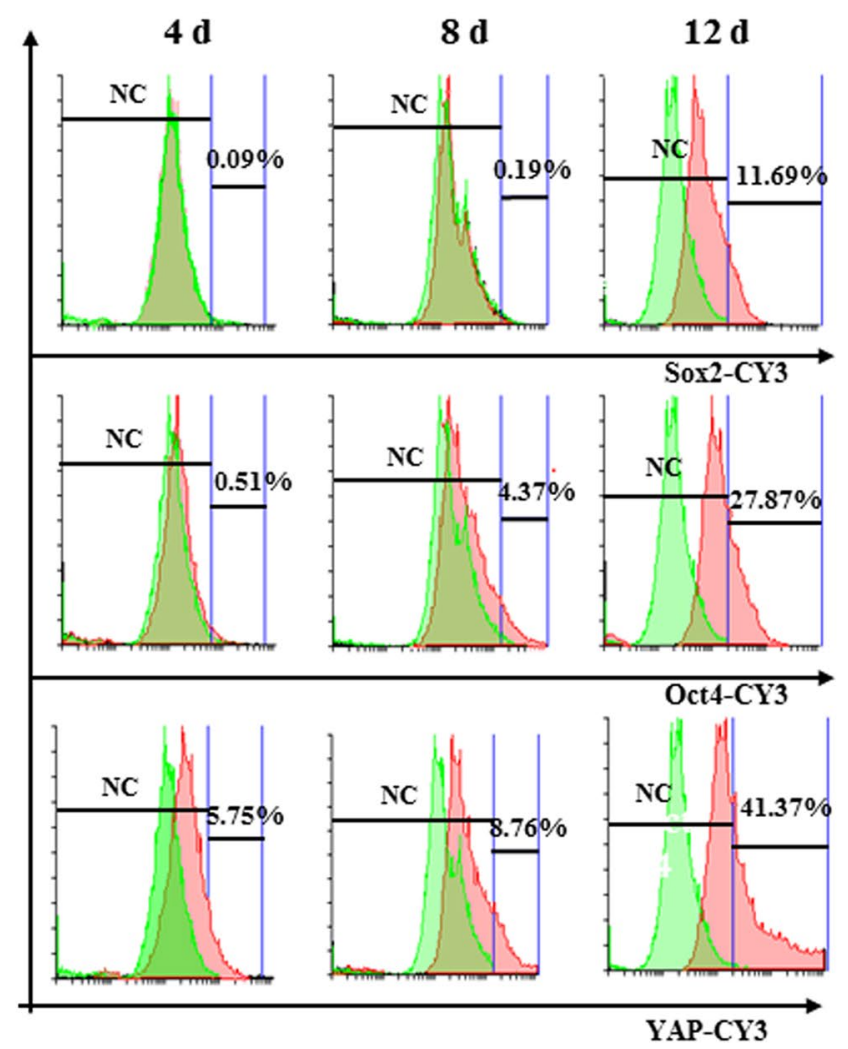

d

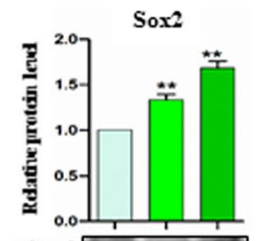

Sox 2

Gapdh厄
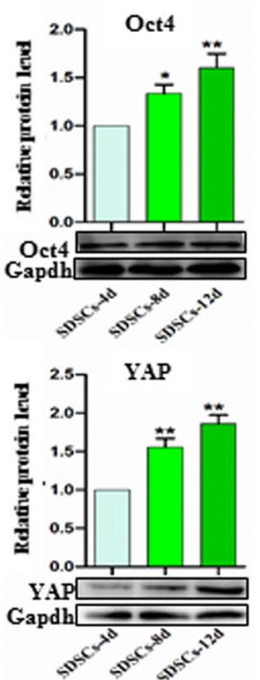

material) and incubated at $4{ }^{\circ} \mathrm{C}$ overnight. In addition, PBS or mouse/rabbit serum was used as primary antibody respectively in negative controls. After the samples were washed three times with PBS, they were incubated for $45 \mathrm{~min}$ at $37{ }^{\circ} \mathrm{C}$ in a moisture chamber with secondary antibody (Table S1 in the supplementary material). The slides were washed three times with PBS and then incubated with Hoechst 33342 to stain the cell nucleus. Finally, the samples were viewed under a laser scanning confocal microscope or fluorescence microscope (Leica TCS-SP5, magnification: objective lense, $\times 20$ and eyepiece, $\times 10$; N.A. (only objective lense): 0.4 , aberration correction: APO, Software: LAS AF2.6.0.7266, Germany) and photographed (Software: LAS AF2.6.0.7266, FITC: excitation wavelength $490 \mathrm{~nm}$ and emission wavelength $525 \mathrm{~nm}$; Cy3: excitation wavelength $550 \mathrm{~nm}$ and emission wavelength $570 \mathrm{~nm}$ ). 


\section{Fluorescence activated cell sorting (FACS) analysis}

The prepared pSDSCs $\left(1 \times 10^{7}\right.$ cells $\left./ \mathrm{ml}\right)$ were fixed in $80 \%$ methanol. The pSDSCs were incubated successively with primary antibodies and secondary antibody (Table $\mathrm{S} 1$ in the supplementary material) at $37^{\circ} \mathrm{C}$ for $60 \mathrm{~min}$. The cells were centrifuged and then analyzed by flow cytometry (Becton Dickinson, Mountain View, CA). Data were analyzed using WinMDI 2.9 software.

\section{Small interfering RNA (siRNA) transfection}

Three fluorescein-labeled siRNA targeted to YAP (siYAP1, siYAP-2, siYAP-3) and fluorescein-labeled non-sense siRNA (siYAP-negative control, siYAP-NC) were purchased form GenePharma Co., Ltd. (Shanghai, China) (Table S2 in the supplementary material). Each siRNA was transfected at a concentration of $30 \mathrm{pmol}$ into the cells using Lipofectamine 2000 (11,668-019; Invitrogen, Carlsbad, CA) following the manufacturer's protocol and viewed and photographed through an inverted microscope (Nikon TE$2000 U$, magnification: objective lense, $\times 10$; eyepiece, $\times 10$; N.A. (only objective lense): 0.25 , camera: DS-U2 (12), Software: NIS-Element 3.0, Japan). The pSDSCs were collected at $48 \mathrm{~h}$ post transfection for further experiments. All the siRNA experiments were performed three times.

\section{RNA extraction and RT-qPCR}

As previously described (Yan et al. 2019), the total RNA was extracted from $\mathrm{pSDSCs}$ with the RNA prep pure Micro Kit (Aidlab, RN07, Beijing, China). The cDNA was synthesized by TransScript One-Step gDNA Removal and cDNA Synthesis SuperMix (TransGen, AT311-03, Beijing, China). RTqPCR amplification with specific primers (Table $\mathrm{S} 3$ in the supplementary material) was done using a Light Cycler 480 (Roche, Germany). The relative transcript abundance was calculated by the $2^{-\Delta \Delta \mathrm{Ct}}$ method (Livak and Schmittgen 2001) and normalized according to the housekeeping gene Gapdh.

\section{Western blot analysis}

Proteins were extracted using RIPA buffer (Beyotime, P0013C, Nantong, China). SDS was mixed with the samples and boiled for $5 \mathrm{~min}$ in water to denature proteins. SDSPAGE was then used to separate the proteins. The proteins were transferred onto a polyvinylidene fluoride (PVDF) membrane (Millipore, ISEQ00010, USA), and blocked in TBST containing $6 \%$ BSA for $4 \mathrm{~h}$. The membranes were incubated with primary antibody (Table $\mathrm{S} 1$ in the supplementary material) at $4{ }^{\circ} \mathrm{C}$; in addition, the membranes were also incubated with PBS or mouse/rabbit serum respectively as negative control, and then a horseradish peroxidase (HRP)-conjugated corresponding secondary antibody (Beyotime) was incubated at room temperature for $120 \mathrm{~min}$ on the second day. The BeyoECL Plus Kit (Beyotime, P0018) was used for chemiluminescence detection. The relative expression level of the target protein to GAPDH was calculated using AlphaView SA (ProteinSimple, CA, USA) software.

\section{Statistical analysis}

GraphPad Prism 8 (GraphPad Software, San Diego, CA) was used for data analysis with one-way analysis of variance (ANOVA) followed by Tukey's test for multiple comparisons; $P<0.05$ means statistically significant difference, $P<0.01$ means extremely significant difference, and $P>0.05$ means not significant (ns). The data were expressed as mean \pm standard error (SE) and all the experiments were repeated at least three times.

\section{Results}

\section{Endogenous YAP expression in pSDSCs}

pSDSCs were cultured using a previously described method (Yan et al. 2019). Spherical aggregates of cells were clearly visible by day 8 of culture (Fig. 1a). FACS analysis during the culturing of the pSDSCs showed dynamic marker changes with the percentage positive for Sox 2 being $0.09 \%$ at 4 days, $0.19 \%$ at 8 days, and $11.69 \%$ at 12 days; for Oct 4 , at 4 days $0.51 \%, 8$ days $4.37 \%$, and 12 days $27.87 \%$; for YAP, at 4 days $5.75 \%, 8$ days $8.76 \%, 12$ days $41.37 \%$. Using FACS and western blot analysis revealed that, during the culturing of pSDSCs, the percentage of the pluripotency markers Oct4 and Sox 2 (Estaras et al. 2017) increased dramatically. Concomitant with the increase of stem cell markers, YAP-positive cells were also significantly upregulated (Fig. 1). These results confirmed that YAP expression is increased during the culturing of pSDSCs, suggesting that it may play a role in self-renewal.

\section{LPC activates YAP and promotes pSDSCs pluripotency}

We explored existing data to identify a small molecule that could increase YAP expression. We found that lysophosphatidylcholine (LPC) could be hydrolyzed into lysophosphatidic acid (LPA) by autotaxin (ATX) (Bao et al. 2018) and LPA acts through G-protein-coupled receptors to inhibit the Hippo pathway kinases Lats $1 / 2$, thereby activating YAP which is an oncoprotein repressed by Lats $1 / 2$ (Yu et al. 2012). In order to investigate the potential effects of different concentrations of LPC on regulating 
YAP in pSDSCs, $0,5,10$, and $25 \mu \mathrm{M}$ LPC was added to the pSDSCs medium, respectively. As show in Fig. 2a, after 12 days culture, spherical colonies were observed in all treatments. FACS analysis of the pSDSCs in the various treatments showed that the percentage of Oct4positive cells increased in the LPC-treated groups in a dose-dependent manner up to $10 \mu \mathrm{M}(44.84 \%$ in $5 \mu \mathrm{M}$ LPC, $53.79 \%$ in $10 \mu \mathrm{M} \mathrm{LPC}$, and $41.84 \%$ in $25 \mu \mathrm{M} \mathrm{LPC}$ ) compared to the control group (28.67\%) (Fig. 2b). On the basis of these results, we used $10 \mu \mathrm{M} \mathrm{LPC}$ for the subsequent studies. In agreement with previous findings
(Bao et al. 2018), exposure to LPC led to an increase in the protein levels of YAP in the pSDSCs (Fig 2c, d). These observations demonstrated that YAP overexpression can be induced by supplementing the culture medium with $10 \mu \mathrm{M}$ LPC.

To investigate the potential effects of LPC-induced YAP expression on regulating the self-renewal of pSDSCs, we examined the protein expression levels of Oct4 and Sox2. As shown in Fig. 2d, both Oct 4 and Sox 2 were upregulated, indicating that YAP plays an important role in the self-renewal of pSDSCs.
Fig. 2 LPC increased the expression of YAP and promoted the pluripotency of pSDSCs. a Morphology of pSDSCs cultured with LPC at different concentrations. Scale bar $=100 \mu \mathrm{m}$. b The percentage of cells positive for Oct4 was detected by FACS of pSDSCs cultured with different concentrations of LPC. c Relative mRNA expression levels of ATX, Sox2, and Oct4 in control and $10 \mu \mathrm{M}$ treated LPC groups. d Representative WB and relative densitometric analysis of ATX, YAP, Sox2, Oct4, and $\beta$-catenin in control and $10 \mu \mathrm{M}$ LPC treated groups. e Morphology of pPGCLCs cultured in control and with $10 \mu \mathrm{M} \mathrm{LPC}$ following differentiation. Scale bar $=50 \mu \mathrm{m}$. f Representative WB and relative densitometric analysis of Vasa in control and $10 \mu \mathrm{M}$ LPC groups following differentiation. $\mathbf{g}$ The positive rate of Vasa was detected by FACS of pPGCLCs cultured with and without $10 \mu \mathrm{M}$ LPC following differentiation. The results are presented as mean \pm SD. $* P<0.05$; $* * P<0.01$ $\mathbf{a}$

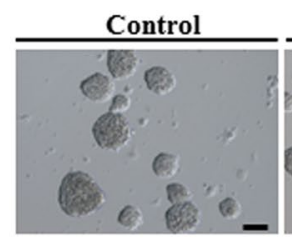

LPC $5 \mu \mathrm{M}$

LPC $10 \mu M$

LPC $25 \mu \mathrm{M}$

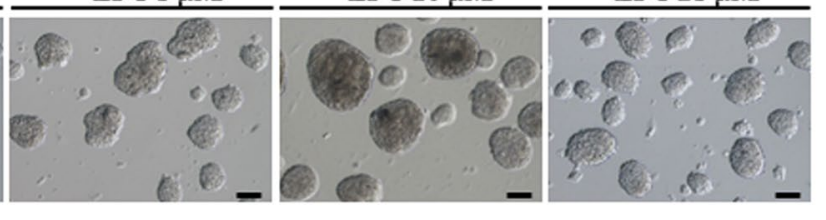

b
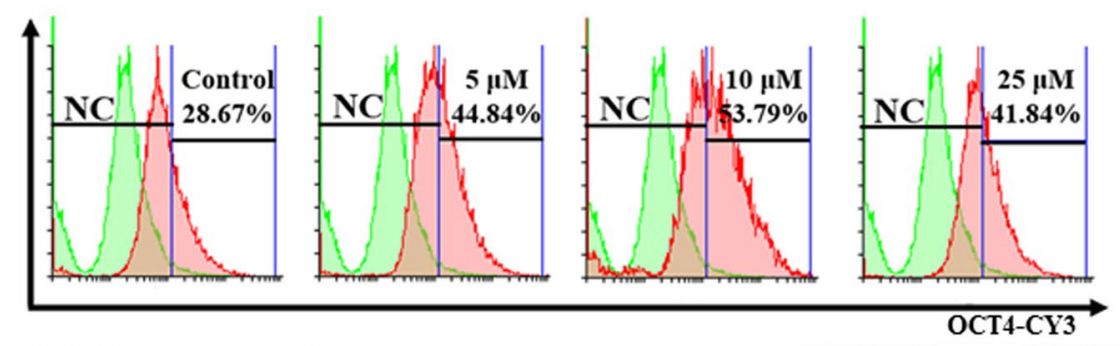

c
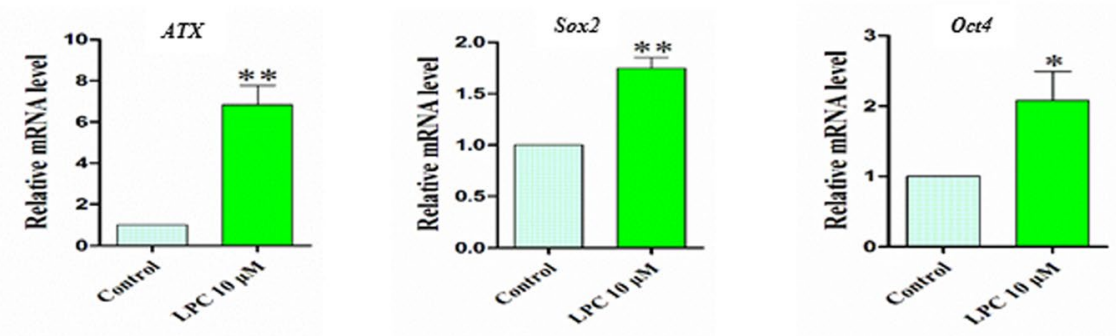

d
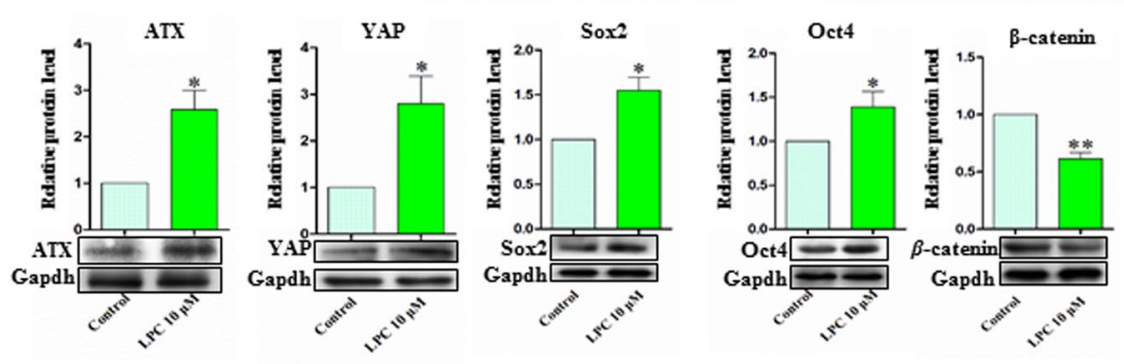

e

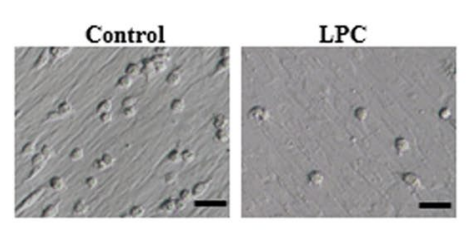

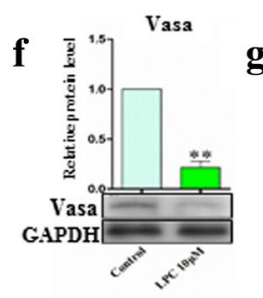

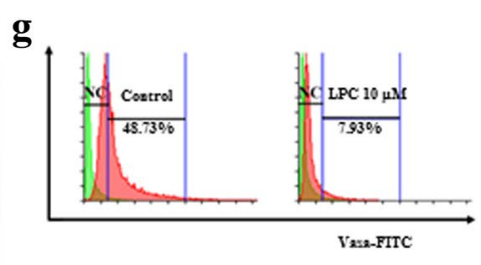




\section{LPC-induced YAP counteracts transdifferentiation of pSDSCs into pPGCLCs}

To evaluated the importance of YAP in the self-renewal of pSDSCs, we examined their ability to differentiate into pPGCLCs in the presence of LPC (Yan et al. 2019; Dyce et al. 2006). The pSDSCs were plated in differentiation medium in 6- $\mathrm{cm}$ dishes, and incubated for 20 days to induce the formation of pPGCLCs. In the untreated control, pSDSCs formed numerous pPGCLCs and high expression of the pPGC marker Vasa (Fig. 2e). In contrast, LPC-treated pSDSCs exhibited resistance to the differentiation and significantly reduced Vasa protein levels under the same differentiation conditions (Fig. 2f). Supporting this, FACS analysis found that the percentage of Vasa-positive cells was $48.73 \%$ in the untreated control and $7.93 \%$ in the LPC treatment group (Fig. 2g). YAP shuttles between the cytoplasm (inactive) and the nucleus (active) (Mo et al. 2014b; Yang et al. 2017). Therefore YAP localization is essential in maintaining the pluripotent properties of stem cells (Mo et al. 2014b; Yang et al. 2017). In our experiments, immunofluorescent staining revealed that YAP was predominantly located in the nucleus of undifferentiated pSDSCs, while significant YAP-positive signals were found in the cytoplasm of differentiated cells (Fig. S1 in the supplementary material). Similar results were found in a previous study using human ESCs (Lian et al. 2010). In conclusion, these results demonstrate that YAP activation maintains pSDSC pluripotency even under differentiation conditions in vitro.

\section{Knockdown of YAP in pSDSCs by siRNA}

To further confirm the role of YAP in pSDSC self-renewal and differentiation, sequence-specific siRNAs against YAP were introduced. Fluorescein-labeled non-sense siRNA ( tions. The expression of the red fluorescent protein was examined by fluorescence microscopy after transfection for 48 h. The results in Fig. 3a demonstrate a successful transfection. In order to further investigate the transfection effect of siRNA, FACS was employed. The results showed that the percentage of CY3-positive cells was similar in the siRNANC and siYAP-1 groups (Fig. 3b). In addition, RT-qPCR and western blot demonstrated that siYAP-1 had the strongest downregulation effect on YAP compared to the other three siRNA groups (Fig. 3c, d).

\section{Knockdown of YAP gene expression suppressed pSDSC pluripotency and promoted pSDSC differentiation}

Striking morphological changes were observed in the siRNA transfected group (Fig. 3e). Knockdown of YAP induced the
pSDSCs cells to acquire a fibroblast-like morphology. The pSDSCs colonies lost their suspended culture and attached and spread on the culture surface. To gain an insight into the molecular mechanisms underlying this morphological phenomenon, the expression levels of two pluripotency markers, Oct4 and Sox2, and a defined terminal differentiation marker involucrin (IVL) (Totaro et al. 2017) were analyzed. As shown in Fig. 3f, YAP knockdown resulted in the downregulation of Oct4 and Sox 2 at the mRNA level and upregulated the expression of IVL. Western blot analysis of protein expression also indicated the same tendencies (Fig. 3g). In addition, the loss of pluripotency in the siYAP transfected pSDSCs was further confirmed by an increase in the percentage of IVL-positive cells using FACS analysis. The results showed that the percentage of IVL-positive cells in the control group was $13.77 \%$, but the percentage in the siYAP treated group was increased to $24.19 \%$ (Fig. $3 \mathrm{~h}$ ).

\section{YAP promotes pSDSC self-renewal by suppressing the Wnt/ $\beta$-catenin signaling pathway}

Previous studies demonstrated that YAP promotes human and mouse stem cell self-renewal in response to suppressing the differentiation-inducing effects of the $\mathrm{Wnt} / \beta$-catenin pathway (Estaras et al. 2017; Lian et al. 2010; Qin et al. 2016). To elucidate the role of YAP in pSDSC self-renewal and its connection with the Wnt/ $\beta$-catenin signaling, we investigated whether YAP regulates $\beta$-catenin activity. Western blot analysis revealed that the protein expression level of $\beta$-catenin was effectively suppressed in LPC-treated pSDSCs (Fig. 2d), while $\beta$-catenin was robustly activated after siYAP-1 transfection (Fig. 3g). These results demonstrate a functional connection between YAP and the $\mathrm{Wnt} / \beta$-catenin signaling pathway.

To further confirm the functional connection between YAP and the Wnt pathway and the role in maintaining the pluripotency of pSDSCs, we assessed the effect of the GSK3 inhibitor CHIR99021 (CHIR), which activates Wnt/ $\beta$ catenin signaling (Estaras et al. 2017), on the differentiation of pSDSCs in LPC treatment. Three dosages $(1 \mu \mathrm{M}, 3 \mu \mathrm{M}$, and $6 \mu \mathrm{M}$ ) of CHIR were used. Interestingly, increasing the concentration of CHIR in pSDSCs induced cells to acquire a flattened phenotype, indicating a loss of characteristic pSDSC morphology (Fig. 4a). FACS analysis indicated that there was a significant upregulation in the percentage of IVL-positive cells in the $3 \mu \mathrm{M}$ and $6 \mu \mathrm{M}$ CHIR-treated groups when compared with the control group (Fig. 4b). In addition, the protein expression levels of $\beta$-catenin and IVL were increased, but both Oct 4 and Sox 2 were significantly decreased and the degrees of reduction of both self-renewal markers were closely correlated with the concentration of CHIR (Fig. 4c). On the basis of the results above, we used $6 \mu \mathrm{M}$ CHIR for subsequent studies. As shown in Fig. 4d, e, 
Fig. 3 siYAP decreased the expression of YAP. a Representative image of pPSDSCs cultured with negative control (NC) and siYAP groups. Scale bar $=50 \mu \mathrm{m}$. b The positive rate of $\mathrm{CY} 3$ was detected by FACS of pSDSCs in control and siYAP-1 groups. c Relative mRNA expression level of $Y A P$ in control, $\mathrm{NC}$, and three YAP siRNAs groups. $\mathbf{d}$ Representative WB and relative densitometric analysis of YAP in control, $\mathrm{NC}$, and three YAP siRNAs groups. e Morphology of pSDSCs with and without the knockdown of YAP. Scale bar $=100 \mu \mathrm{m}$. f Relative mRNA expression level of $I V L$, Sox 2 , and Oct4 in control and siYAP groups. g Representative WB and relative densitometric analysis of IVL, Sox2, Oct4, and $\beta$-catenin in control and siYAP groups. $\mathbf{h}$ The positive rate of IVL was detected by FACS of pPSDSCs in the control, NC, and siYAP groups. The results are presented as mean $\pm \mathrm{SD}$. $* P<0.05 ; * * P<0.01$ $\mathbf{a}$
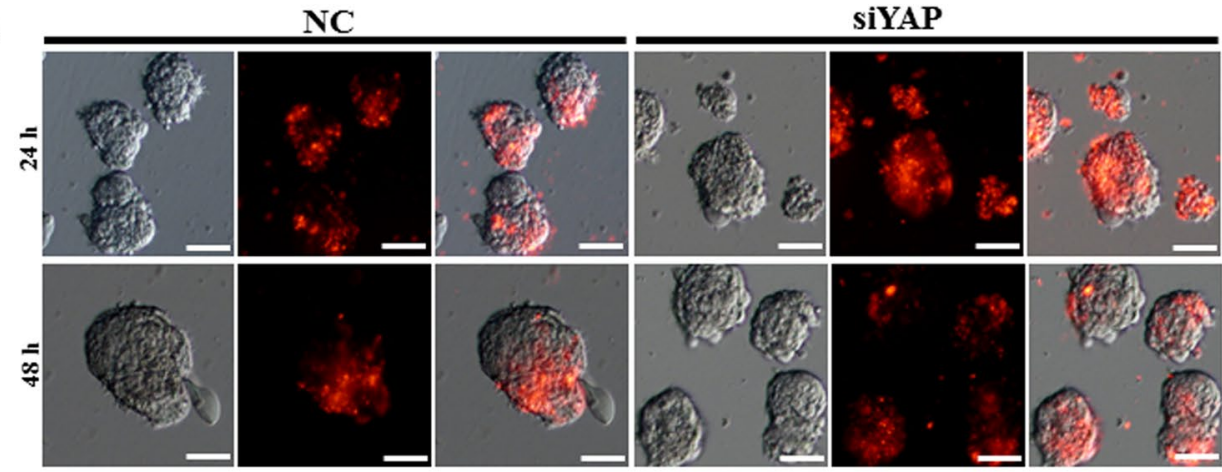

b

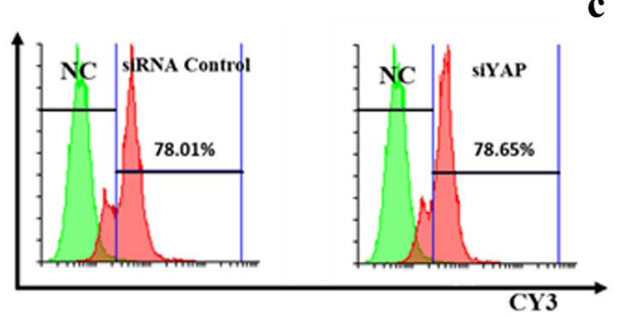

c

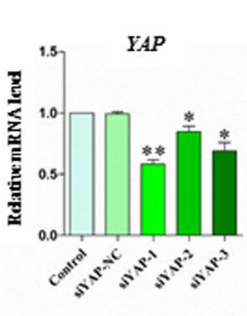

d

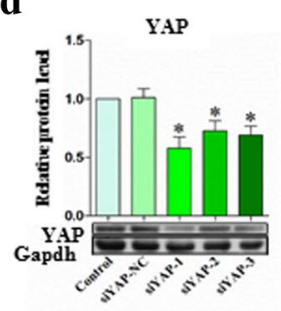

e

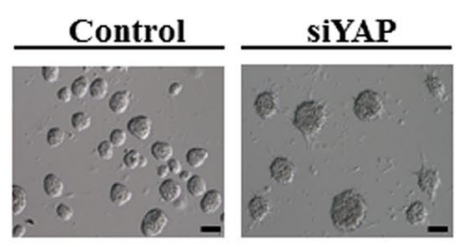

h

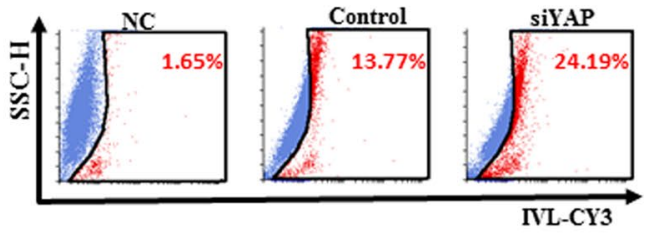

f
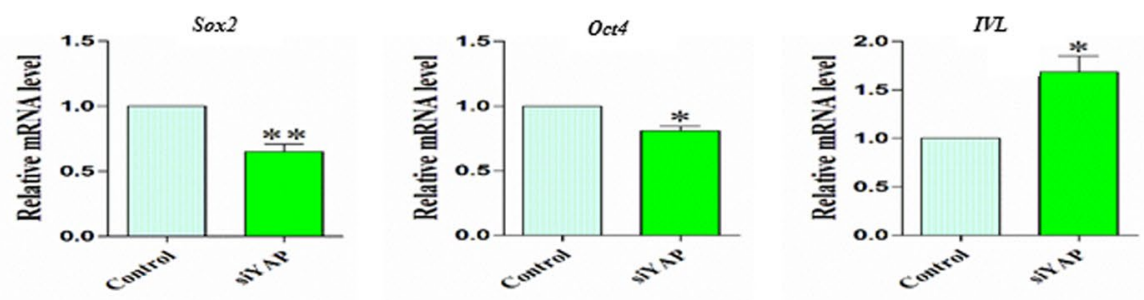

g
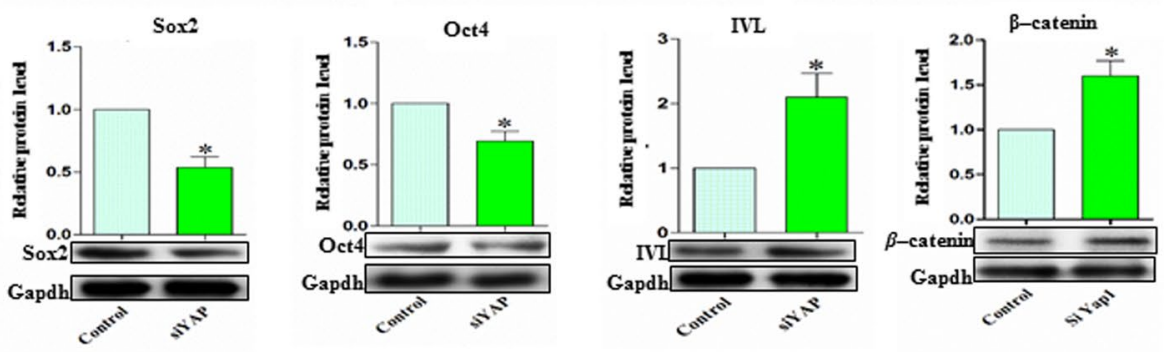

the Wnt pathway was effectively repressed in the $10 \mu \mathrm{M}$ LPC-treated pSDSCs (L-pSDSCs). Upon treating L-pSDSCs with $6 \mu \mathrm{M}$ CHIR, the expression of $\beta$-catenin increased but Oct 4 and Sox 2 decreased (Fig. 4d, e). These results show that activating $\mathrm{Wnt} / \beta$-catenin signaling partially restores the differentiation ability of the L-pSDSC group, as evidenced by the western blot results showing higher expression of IVL (Fig. 4e). Therefore, the results investigating the addition with CHIR imply that YAP promotes pSDSCs self-renewal by suppressing the $\mathrm{Wnt} / \beta$-catenin signaling pathway.

\section{YAP prevents pSDSC differentiation by modulating Wnt/ $\beta$-catenin signaling}

In order to further elucidate the mechanism by which YAP regulates pSDSC differentiation through Wnt signaling, we investigated the effect of XAV939, a Wnt signaling 
Fig. 4 Analysis of pSDSCs treated with CHIR99021.

a Morphology of pSDSCs cultured with different concentrations of CHIR. Scale bar $=100 \mu \mathrm{m}$. b The positive rate of IVL was detected by FACS of pSDSCs cultured with different concentrations of CHIR. c Representative WB and relative densitometric analysis of $\beta$-catenin, IVL, Sox 2 , and Oct 4 of pSDSCs cultured with different concentrations of CHIR. d Relative mRNA expression level of $I V L$, Sox2, and Oct4 in control, LPC, and LPC + CHIR groups. e Representative WB and relative densitometric analysis of $\beta$-catenin, IVL, Sox2, and Oct4 in control, LPC, and LPC + CHIR groups.

The results are presented as mean $\pm \mathrm{SD} . * P<0.05$; $* * P<0.01$ $\mathbf{a}$

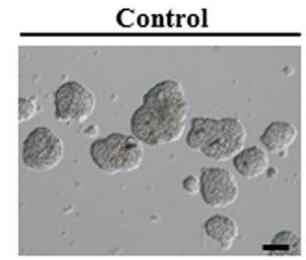

b
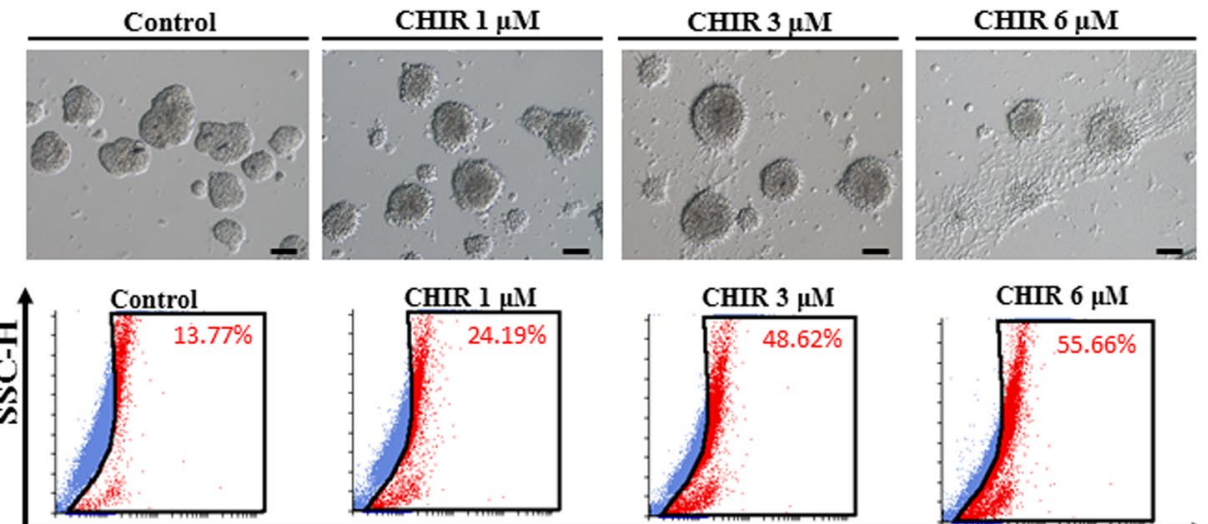

CHIR $3 \mu \mathrm{M}$

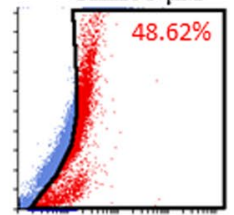

c

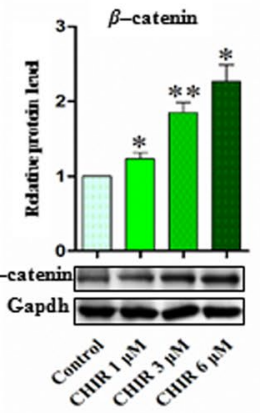

d
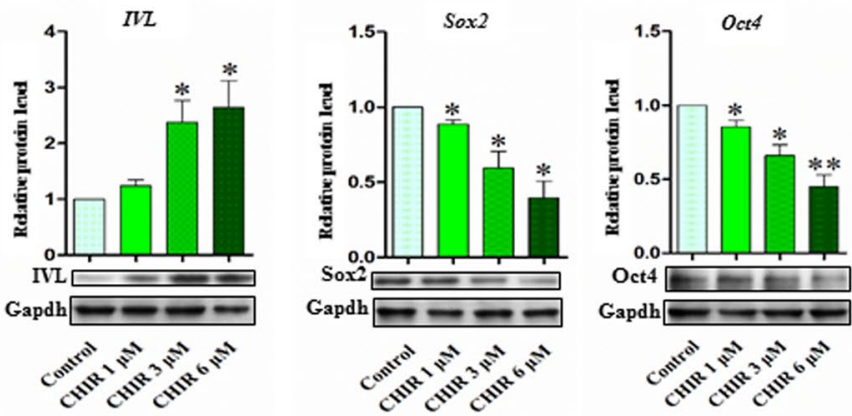

IVL-CY3
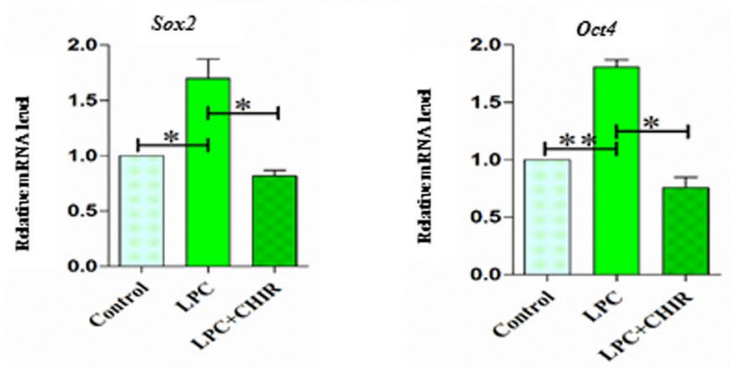

e
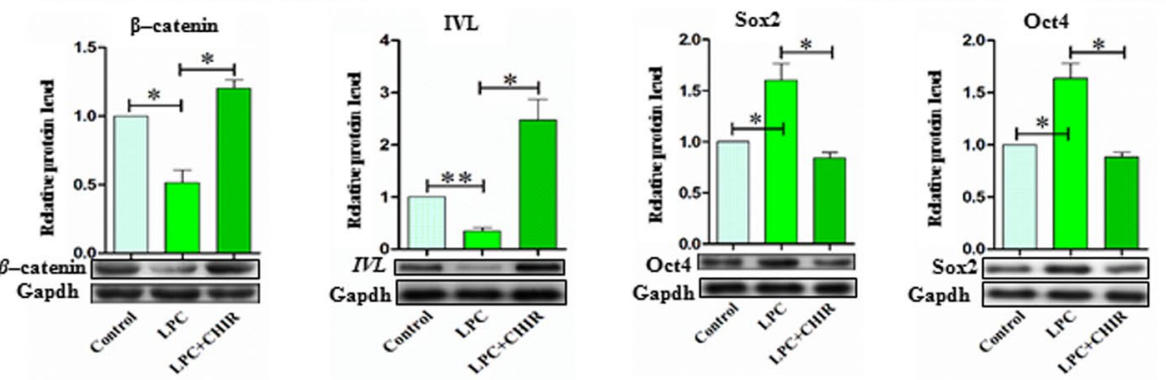

antagonist, on the self-renewal of pSDSCs following YAP knockdown. Three dosages of XAV939 were tested (1 $\mu \mathrm{M}, 5 \mu \mathrm{M}$, and $10 \mu \mathrm{M})$. With increasing concentration of XAV939, the pSDSCs showed an elevated clonal growth rate, and propagated readily in culture for more than five passages (data not show). Western blot analysis indicated that $\beta$-catenin protein was effectively suppressed in the $10 \mu \mathrm{M}$ XAV939-treated groups but Oct4 and Sox 2 were markedly increased (Fig. 5a). Moreover, FACS analysis showed that the percentage of Oct4-positive cells was significantly upregulated in the $10 \mu \mathrm{M}$ XAV939 treatment group when compared with the control group (Fig. 5b). Next, using the siRNA system, we evaluated how YAP modulates pSDSCs self-renewal in the $10 \mu \mathrm{M}$ XAV939-treated cells. The results indicated that siYAP led to increased expression of $\beta$-catenin in the pSDSCs but in the siYAP plus XAV939 group, $\beta$-catenin returned to the control levels (Fig. 5c). These results indicated that the Wnt pathway is important for the differentiation of 
Fig. 5 Analysis of pSDSCs treated with XAV939. a Representative WB and relative densitometric analysis of $\beta$-catenin, Sox 2 , and Oct 4 in pSDSCs cultured with different concentrations of XAV939. b The positive rate of Oct4 was detected by FACS of pPSDSCs cultured with different concentrations of XAV939. c Representative WB and relative densitometric analysis of $\beta$-catenin, Sox 2 , and Oct4 of pSDSCs in control, siYAP, and siYAP + XAV groups. The results are presented as mean \pm SD. $* P<0.05$; $* * P<0.01$
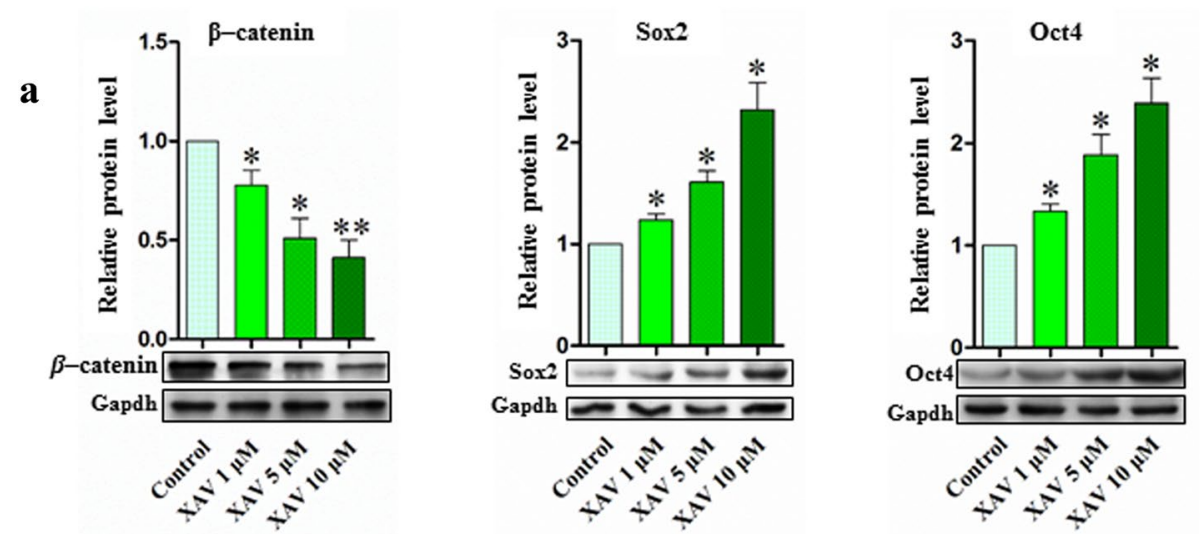

b
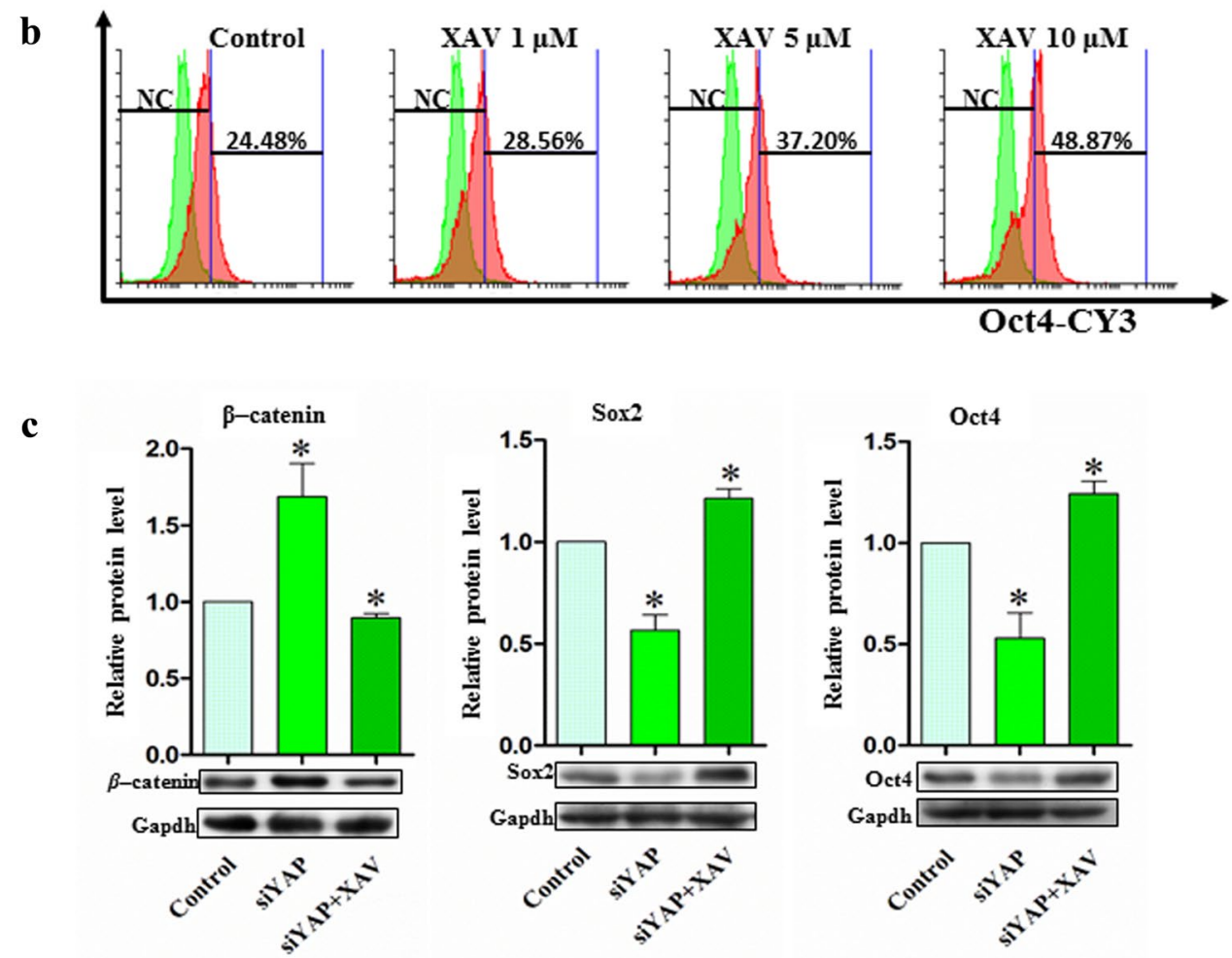

pSDSCs. Moreover, it appears that YAP inhibits pSDSC differentiation in response to $\mathrm{Wnt} / \beta$-catenin signaling.

\section{Discussion}

In this paper, we confirmed that YAP helped maintain the ability of pSDSCs to self-renew through maintaining the high expression of the pluripotency genes Sox 2 and Oct 4 and meanwhile suppressing the $\mathrm{Wnt} / \beta$-catenin signaling pathway. Our observations demonstrated that concomitantly with the increase of YAP-positive cells, the protein levels of Oct4 and Sox 2 were also significantly upregulated (Fig. 2). On the other hand, YAP knockdown leads to a loss of pluripotency in pSDSCs (Fig. 3). Interestingly, higher expression of YAP prevented pSDSC differentiation in vitro even under differentiation-inducing conditions (Fig. 2e-g). Furthermore, we found evidence that the Wnt pathway regulates pSDSC differentiation. Our experiments indicated that the addition of CHIR, an activator of the Wnt/ $\beta$-catenin signaling pathway, in pSDSCs medium results in a rapid loss of stem cell properties, including morphological changes and decreased expression of the pluripotency markers, Oct 4 and Sox 2 (Fig. 4a). Conversely, when the Wnt/ $\beta$-catenin signaling pathway is inhibited using XAV939, we confirmed that stem cell markers were positively upregulated (Fig. 5a, b). We also found evidence supporting a relationship between YAP and the $\mathrm{Wnt} / \beta$-catenin signaling pathway. YAP overexpression or knockdown could affect the protein expression level of $\beta$-catenin, a core component of the Wnt pathway, 
and thereby inhibit or promote the differentiation of pSDSC. The results in Fig. 4d, e support that pSDSCs treated with LPC plus CHIR resulted in increased protein levels of $\beta$-catenin and IVL and decreased protein levels of Oct 4 and Sox 2 compared with that of LPC treatment alone. In addition, we also confirmed that knockdown of YAP induced the differentiation of pSDSCs, but treatment with XAV939 after YAP knockdown prevented this differentiation (Fig. 5c). To summarize, these observations mutually corroborate each other and further establish that YAP plays an important role in maintaining the pluripotency of pSDSCs partly through repressing the $\mathrm{Wnt} / \beta$-catenin signaling pathway.

Recent studies have shown that core transcription factors, such as Oct4, Sox2, and Nanog, play an essential role in establishing and controlling gene expression programs which define the identity of human and mouse embryonic stem cells (ESCs) (Boyer et al. 2005). In particular, these core transcription factors are important for establishing the ability of ESCs and ASCs to self-renew and differentiate (Kim et al. 2008; Chambers and Smith 2004; Seo et al. 2013). Several studies have presented data showing that YAP can facilitate self-renewal through the upregulation of core transcription factors, such as Oct4, Sox2, and Nanog, and inhibiting YAP function results in the differentiation of ESCs (Tamm et al. 2011; Mo et al. 2014b; Lian et al. 2010). In addition, on the basis of transcriptional profiling, Ramalho-Santos et al. (2002) demonstrated that YAP also has a critical role in the self-renewing ability of ASCs, such as neural stem cells and hematopoietic stem cells. These results strongly support our work; for instance, in this paper self-renewal of the pSDSCs was identified on the basis of the expression level of protein markers, Oct4, Sox2, and Nanog, and our results showed a striking correlation between the protein expression level of YAP and that of Oct4 and Sox2 (Fig. 1). YAP was highly expressed in self-renewing pSDSCs and was downregulated when cells were induced to differentiate. Meanwhile, studies described in this manuscript revealed that overexpression of YAP prevented the differentiation of pSDSCs (Fig. 2) and the depletion of YAP by siRNAs suppressed the self-renewal of pSDSCs (Fig. 3). These observations strongly confirm a particularly important role for YAP in maintaining the "stemness" of pSDSCs.

Genome-wide analysis of mouse ESCs by Lian et al. (2010) showed that YAP can positively regulate pluripotent genes such as Nanog, Oct4, and Sox2. Bora-Singhal et al. (2015) further found that the structural domain of the YAP protein can interact with the transcription factor Oct4 to induce the transcriptional activity of Sox2-regulated downstream targets and maintain the self-renewal ability of stem cells. Reciprocally, Seo et al. (2013) showed that YAP expression is regulated by Sox 2 and could compensate for the self-renewal defect caused by Sox 2 inactivation in mesenchymal stem cells (MSCs). Given these facts, the relationship between YAP and Oct4 or Sox 2 is rather interesting and complex and requires further investigation.

Extensive cross talk between YAP and $\beta$-catenin, a key protein of the Wnt signaling pathway, has been observed (Imajo et al. 2012; Seo et al. 2013; Qin et al. 2016; Pan et al. 2018). Qin et al. (2016) confirmed that YAP promotes naive pluripotency of human ESCs by interfering with $\beta$-catenin and retaining it in the cytoplasm to suppress Wnt signaling. Seo et al. (2013) found that in MSCs, YAP binds $\beta$-catenin and inhibits canonical Wnt signaling to prevent MSC differentiation. Our results are in line with these previous findings; after overexpression of YAP, the expression of $\beta$-catenin was decreased (Fig. 2c, d). Conversely, the expression of $\beta$-catenin increased when we knock down YAP using RNAi (Fig. 3f, g). Similarly, when we added CHIR to the LPCtreated pSDSCs we found that the differentiation ability of pSDSCs was partially restored through activation of the Wnt pathway (Fig. 4d, e). This suggests that YAP was sufficient to maintain the self-renewal characteristics of pSDSCs by inhibiting the Wnt pathway. In order to more clearly investigate the mechanism of YAP regulating the self-renewal of pSDSCs through the Wnt/ $\beta$-catenin signaling pathway, we added the inhibitor of the Wnt pathway XAV939 to the pSDSCs that had YAP knocked down. With the addition of XAV939, we found that the cell fate of pSDSCs was altered. Compared with pSDSCs with low YAP, the addition of XAV939 resulted in pSDSCs with higher self-renewal ability (Fig. 5). Taken together, this supports YAP regulating the fate of pSDSCs though its effects on the $\mathrm{Wnt} / \beta$-catenin signaling pathway (Fig. 6).

In conclusion, we have shown that YAP regulates the self-renewal of pSDSCs by maintaining the high expression of the pluripotency genes Sox 2 and Oct4 and meanwhile suppressing the Wnt pathway. However, the specific processes and mechanisms of the interaction between the Hippo and Wnt pathways are not fully understood. Further study of the interactions between the members of these

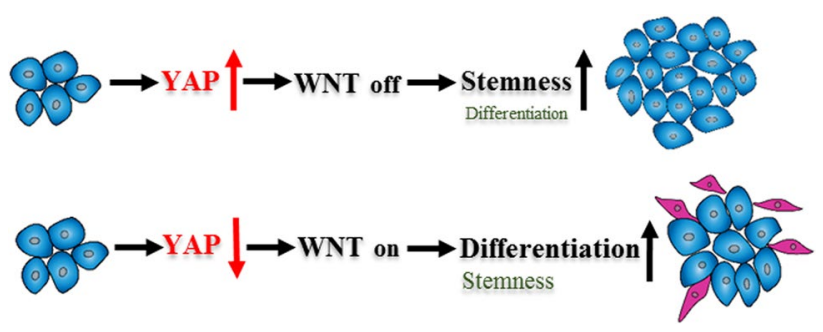

Fig. 6 Schematic model for the mechanism of YAP regulating the fate of pSDSCs is related to the $\mathrm{Wnt} / \beta$-catenin pathway. Overexpression of YAP promotes pSDSCs self-renewal by suppressing the Wnt/ $\beta$-catenin signaling pathway. Knockdown of YAP induces the differentiation of pSDSCs through activating the $\mathrm{Wnt} / \beta$-catenin signaling pathway 
two signaling pathways is expected to provide new insights into the molecular basis of many biological processes.

Supplementary Information The online version contains supplementary material available at https://doi.org/10.1007/s00418-021-02034-4.

Acknowledgements This work was supported by National Natural Science Foundation of China (32072734, 31672423), Shandong Province Key Research and Development Program (2019GSF107082) and Taishan Scholar Construction Foundation of Shandong Province (ts20190946).

Author contributions WS and SFC conceived the study; HCY, YS, MYZ, and JDS carried out experiments and data analysis; SEZ, PWD, FGK, and MDF interpreted the data and wrote the manuscript. All authors approved the final version. Hong-Chen Yan: Formal analysis, Investigation. Yu Sun: Investigation. Ming-Yu Zhang: Writing-Original Draft. Shu-Er Zhang: Investigation. Jia-Dong Sun: Investigation. Paul W. Dyce: Writing-Review \& Editing. Francesca Gioia Klinger: Writing-Review \& Editing. Massimo De Felici: Writing-Review \& Editing. Wei Shen: Writing—review \& editing, Project administration. Shun-Feng Cheng: Writing-Original Draft, Project administration.

\section{Declarations}

Conflict of interest The authors declare that there is no conflict of interest that could be perceived as prejudicing the impartiality of the research reported.

\section{References}

Bao L, Qi J, Wang YW, Xi Q, Tserennadmid T, Zhao PF, Qi J, Damirin A (2018) The atherogenic actions of LPC on vascular smooth muscle cells and its LPA receptor mediated mechanism. Biochem Biophys Res Commun 503(3):1911-1918. https://doi. org/10.1016/j.bbrc.2018.07.135

Bora-Singhal N, Nguyen J, Schaal C, Perumal D, Singh S, Coppola D, Chellappan S (2015) YAP 1 regulates OCT 4 activity and SOX 2 expression to facilitate self-renewal and vascular mimicry of stem-like cells. Stem Cells 33(6):1705-1718

Boyer LA, Lee TI, Cole MF, Johnstone SE, Levine SS, Zucker JP, Guenther MG, Kumar RM, Murray HL, Jenner RG, Gifford DK, Melton DA, Jaenisch R, Young RA (2005) Core transcriptional regulatory circuitry in human embryonic stem cells. Cell 122(6):947-956. https://doi.org/10.1016/j.cell.2005.08.020

Chambers I, Smith A (2004) Self-renewal of teratocarcinoma and embryonic stem cells. Oncogene 23(43):7150-7160. https://doi. org/10.1038/sj.onc. 1207930

Dyce PW, Wen L, Li J (2006) In vitro germline potential of stem cells derived from fetal porcine skin. Nat Cell Biol 8(4):384-390

Estaras C, Hsu HT, Huang L, Jones KA (2017) YAP repression of the WNT3 gene controls hESC differentiation along the cardiac mesoderm lineage. Genes Dev 31(22):2250-2263. https://doi. org/10.1101/gad.307512.117

Fuchs E, Chen T (2013) A matter of life and death: self-renewal in stem cells. EMBO Rep 14(1):39-48

Ge W, Ma H-G, Cheng S-F, Sun Y-C, Sun L-L, Sun X-F, Li L, Dyce P, Li J, Shi Q-H (2015) Differentiation of early germ cells from human skin-derived stem cells without exogenous gene integration. Sci Rep 5(1):1-9
Ge W, Cheng S-F, Dyce PW, De Felici M, Shen W (2016) Skinderived stem cells as a source of primordial germ cell-and oocyte-like cells. Cell Death Dis 7(11):e2471-e2471

Hayashi K, Ogushi S, Kurimoto K, Shimamoto S, Ohta H, Saitou M (2012) Offspring from oocytes derived from in vitro primordial germ cell-like cells in mice. Science 338(6109):971-975

Huang S-MA, Mishina YM, Liu S, Cheung A, Stegmeier F, Michaud GA, Charlat O, Wiellette E, Zhang Y, Wiessner S (2009) Tankyrase inhibition stabilizes axin and antagonizes Wnt signalling. Nature 461(7264):614-620

Imajo M, Miyatake K, Iimura A, Miyamoto A, Nishida E (2012) A molecular mechanism that links Hippo signalling to the inhibition of Wnt/ $\beta$-catenin signalling. EMBO J 31(5):1109-1122

Jeong S-H, Kim H-B, Kim M-C, Lee J-m, Lee JH, Kim J-H, Kim J-W, Park W-Y, Kim S-Y, Kim JB (2018) Hippo-mediated suppression of IRS2/AKT signaling prevents hepatic steatosis and liver cancer. J Clin Inves 128(3):1010-1025

Judson RN, Tremblay AM, Knopp P, White RB, Urcia R, De Bari C, Zammit PS, Camargo FD, Wackerhage H (2012) The Hippo pathway member Yap plays a key role in influencing fate decisions in muscle satellite cells. J Cell Sci 125(24):6009-6019

Kim J, Chu J, Shen X, Wang J, Orkin SH (2008) An extended transcriptional network for pluripotency of embryonic stem cells. Cell 132(6):1049-1061. https://doi.org/10.1016/j.cell.2008.02. 039

Kobayashi T, Zhang H, Tang WWC, Irie N, Withey S, Klisch D, Sybirna A, Dietmann S, Contreras DA, Webb R, Allegrucci C, Alberio R, Surani MA (2017) Principles of early human development and germ cell program from conserved model systems. Nature 546(7658):416-420. https://doi.org/10.1038/nature22812

Lee JH, Kim TS, Yang TH, Koo BK, Oh SP, Lee KP, Oh HJ, Lee SH, Kong YY, Kim JM (2008) A crucial role of WW45 in developing epithelial tissues in the mouse. EMBO J 27(8):1231-1242

Lian I, Kim J, Okazawa H, Zhao J, Zhao B, Yu J, Chinnaiyan A, Israel MA, Goldstein LS, Abujarour R, Ding S, Guan KL (2010) The role of YAP transcription coactivator in regulating stem cell selfrenewal and differentiation. Genes Dev 24(11):1106-1118. https:// doi.org/10.1101/gad.1903310

Linher K, Dyce P, Li J (2009) Primordial germ cell-like cells differentiated in vitro from skin-derived stem cells. PLoS ONE 4(12):e8263. https://doi.org/10.1371/journal.pone.0008263

Livak KJ, Schmittgen TD (2001) Analysis of relative gene expression data using real-time quantitative PCR and the 2(-Delta Delta C(T)) method. Methods 25(4):402-408. https://doi.org/10.1006/ meth.2001.1262

Mo JS, Park HW, Guan KL (2014a) The Hippo signaling pathway in stem cell biology and cancer. EMBO Rep 15(6):642-656

Murillo-Garzón V, Kypta R (2017) WNT signalling in prostate cancer. Nat Rev Urol 14(11):683

Pan J-X, Xiong L, Zhao K, Zeng P, Wang B, Tang F-L, Sun D, Guo $\mathrm{H}-\mathrm{h}$, Yang X, Cui S (2018) YAP promotes osteogenesis and suppresses adipogenic differentiation by regulating $\beta$-catenin signaling. Bone Res 6(1):1-12

Piccolo S, Dupont S, Cordenonsi M (2014) The biology of YAP/TAZ: hippo signaling and beyond. Physiol Rev 94(4):1287-1312

Qin H, Hejna M, Liu Y, Percharde M, Wossidlo M, Blouin L, Durruthy-Durruthy J, Wong P, Qi Z, Yu J (2016) YAP induces human naive pluripotency. Cell Rep 14(10):2301-2312

Ramalho-Santos M, Yoon S, Matsuzaki Y, Mulligan RC, Melton DA (2002) "Stemness": transcriptional profiling of embryonic and adult stem cells. Science 298(5593):597-600. https://doi.org/10. 1126/science. 1072530

Seo E, Basu-Roy U, Gunaratne PH, Coarfa C, Lim DS, Basilico C, Mansukhani A (2013) SOX2 regulates YAP1 to maintain stemness and determine cell fate in the osteo-adipo lineage. Cell Rep 3(6):2075-2087. https://doi.org/10.1016/j.celrep.2013.05.029 
Singh MK, Mia MM (2019) The Hippo signaling pathway in cardiac development and diseases. Front Cell Dev Biol 7:211

Tamm C, Böwer N, Annerén C (2011) Regulation of mouse embryonic stem cell self-renewal by a Yes-YAP-TEAD2 signaling pathway downstream of LIF. J Cell Sci 124(7):1136-1144

Totaro A, Castellan M, Battilana G, Zanconato F, Azzolin L, Giulitti S, Cordenonsi M, Piccolo S (2017) YAP/TAZ link cell mechanics to Notch signalling to control epidermal stem cell fate. Nat Commun 8:15206. https://doi.org/10.1038/ncomms15206

Xin M, Kim Y, Sutherland LB, Murakami M, Qi X, McAnally J, Porrello ER, Mahmoud AI, Tan W, Shelton JM (2013) Hippo pathway effector Yap promotes cardiac regeneration. Proc Natl Acad Sci 110(34):13839-13844

Yan HC, Li L, Liu JC, Wang YF, Liu XL, Ge W, Dyce PW, Li L, Sun XF, Shen W, Cheng SF (2019) RA promotes proliferation of primordial germ cell-like cells differentiated from porcine skinderived stem cells. J Cell Physiol 234(10):18214-18229. https:// doi.org/10.1002/jcp. 28454

Yang B, Sun H, Song F, Yu M, Wu Y, Wang J (2017) YAP1 negatively regulates chondrocyte differentiation partly by activating the beta-catenin signaling pathway. Int J Biochem Cell Biol 87:104113. https://doi.org/10.1016/j.biocel.2017.04.007

Yu FX, Zhao B, Panupinthu N, Jewell JL, Lian I, Wang LH, Zhao J, Yuan H, Tumaneng K, Li H, Fu XD, Mills GB, Guan KL (2012) Regulation of the Hippo-YAP pathway by G-protein-coupled receptor signaling. Cell 150(4):780-791. https://doi.org/10.1016/j. cell.2012.06.037

Zhang H, Pasolli HA, Fuchs E (2011) Yes-associated protein (YAP) transcriptional coactivator functions in balancing growth and differentiation in skin. Proc Natl Acad Sci 108(6):2270-2275

Zhao B, Tumaneng K, Guan K-L (2011) The Hippo pathway in organ size control, tissue regeneration and stem cell self-renewal. Nat Cell Bio 13(8):877-883

Publisher's Note Springer Nature remains neutral with regard to jurisdictional claims in published maps and institutional affiliations. 